\title{
Cooperative fluctuations of PTP1B by an elastic network model analysis
}

\section{AC Serdaroglu $₫$, AN Ozer}

Department of Bioengineering, Marmara University, Istanbul

\section{Motivations}

Protein tyrosine phosphorylation is essential in controlling many vital activities of the cell such as growth, differentiation, metabolism and immune response. Abnormal tyrosine phosphorylation leads to various human diseases including cancers, diabetes, rheumatoid arthritis and hypertension. PTP1B, which is a major negative regulator of insulin signaling, is one of the important forms of tyrosine specific phosphatases that hydrolyze phosphotyrosine containing proteins. As the loss of PTPIB activity leads to enhanced insulin sensitivity and resistance to weight gain, inhibiting PTPIB activity represents a novel approach for the treatment of diabetes and obesity. In this regard, understanding the molecular recognition mechanism in binding processes of PTPIB may provide guidelines for the development of potent PTP1B inhibitors.

\section{Methods}

Here, the structural dynamics of both substrateand inhibitor-bound PTPIB are studied comparatively using the Anisotropic Network Model (ANM) which performs harmonic vibrational analysis around the equilibrium states and predicts the directionalities of the collective motions as well as their magnitudes.

\section{Results}

The mean-square fluctuations in the most cooperative ANM modes are similar in different PTPIB complex structures and the minimum fluctuating residues correspond to the dynamically correlated hinge regions. Further, the variation in the orientation of the fluctuations is caused mainly by the residues lying along the rotational axes that are responsible for the functional motions. Overall, the elaborated analysis of the structural fluctuations of PTPIB in interaction with its ligands helps to gain insight into the dynamics of the phosphatase in relation to its function. 